\title{
On Recursive Refinement of Convex Polygons
}

\author{
Ming-Jun Lai* and George Slavov ${ }^{\dagger}$ \\ Department of Mathematics \\ The University of Georgia \\ Athens, GA 30602
}

March 2, 2016

\begin{abstract}
It is known that one can improve the accuracy of the finite element solution of partial differential equations (PDE) by uniformly refining a triangulation. Similarly, one can uniformly refine a quadrangulation. Recently polygonal meshes have been used for numerical solution of partial differential equations based on virtual element methods, weak Galerkin methods, and polygonal spline methods. A refinement scheme of pentagonal partition was introduced in [6]. It is natural to ask if one can create a hexagonal refinement or general polygonal refinement schemes. In this short article, we show that one cannot refine a convex hexagon using convex hexagons of smaller size. In general, we show that one can only refine a convex n-gon by convex n-gons of smaller size if $n \leq 5$.
\end{abstract}

\section{Introduction}

In the fields of computer aided geometry design of surfaces and numerical solutions of partial differential equations (PDE), triangulations have been the traditional way of partitioning spatial domains. Due to the recent development of the virtual element methods, weak Galerkin methods, and polygonal splines (see [2], [3], [12], [13], [15], 6]), one is able to use an arbitrary polygonal partition for numerical solutions of PDE. In addition, generalized barycentric coordinates (GBC) over arbitrary polygons of $n$ sides, $n$-gon for short, were invented for surface applications. See a recent survey in [5]. An excellent polygonal mesh generator can be found in [14. It is known that we can uniformly refine a triangulation and a quadrangulation (cf. [11]) which is a common strategy to demonstrate the accuracy as well as the convergence of a numerical algorithm for solving a PDE. Recall the standard theory of spline approximation (cf. e.g.[11]) and the finite element method (cf. e.g. [4]), i.e., the h-version and hp-version of finite element method requires the size of a underlying partition determines go to zero. It is important to have a scheme to generate partitions with finer sizes. Refining an existing partition to a partition of the same type with smaller size is an obvious approach which can be conveniently applied repeatedly to reduce the size of underlying partition. In addition, for polynomial finite elements or bivariate splines (cf. [1]), the uniform refinement of triangulations/quadrangulations enables the spline spaces to have the nestedness property of the function spaces which can be important for several applications, e.g. construction of a multi-resolution analysis which leads to wavelets or tight wavelet frames (cf. e.g. [7]) as well as construction of multi-grid

*mjlai@math.uga.edu. This research is partially supported by Simons collaboration grant 280646 and the National Science Foundation under the grant \#DMS 1521537.

†gpslavov@uga.edu 
methods for numerical solutions of PDE (cf. e.g. [4]). Another important feature of uniformly refining an underlying partition is to make a computer code easy to implement and efficient to run.

Recently a refinement scheme of pentagonal partitions was introduced in [6], pictured in Fig. 1. and used to reduce the error in numerical solutions based on polygonal splines which consist of generalized Bernstein-Bézier functions in terms of GBC.
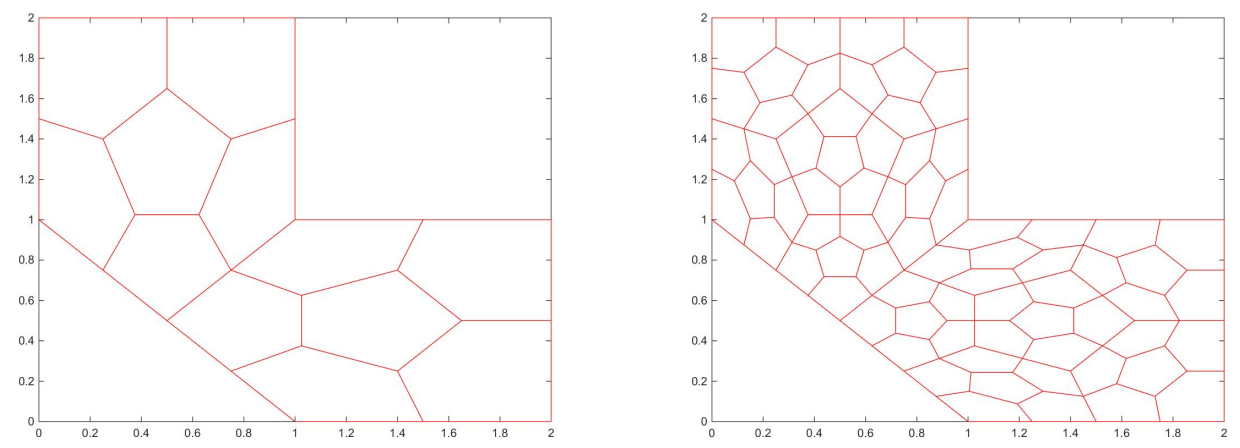

Figure 1: A Pentagonal Partition (left) and Its Refinement (right)

A natural question to ask is if one can create a hexagonal refinement, i.e. refine a convex hexagon by using convex hexagons of smaller size. In general, one can ask if one can create a general polygonal refinement scheme. In this short article, we will show that one cannot refine a convex hexagon by convex hexagons only. In fact, our arguments prove more. That is, one cannot refine a convex n-gon by convex $n$-gons of smaller size whenever $n \geq 6$. Hence, if one uses a polygonal mesh of single polygon type, then one cannot expect to generate the mesh starting from a few seeded convex $n$-gons with $n \geq 6$ by a recursive refinement scheme. This result will be shown in the next section. Then we shall discuss how to refine a general $n$-gon. We introduce a simple remedy refinement scheme of hexagons by using pentagons and one hexagon of smaller size. Similarly, a general convex $n$-gon can be refined by using pentagons and a convex $n$-gon of smaller size. In addition, we shall pose a few open questions about the possibility of refining a domain of general shape by using pentagons only. All these will be contained in $\S 3$.

\section{Main Results and Proofs}

\subsection{Partitions of Polygons}

Definition 2.1. Let $V=\left\{v_{1}, v_{2}, \ldots, v_{n}\right\} \subset \mathbb{R}^{2}$ be a set of points. An edge $e_{k}$ connecting $v_{i_{k}}$ to $v_{j_{k}}$ for some $i_{k}$ and $j_{k}$ in $\{1,2, \ldots, n\}$ is defined as $e_{k}=\left\{x \in \mathbb{R}^{2} \mid x=t v_{i_{k}}+(1-t) v_{j_{k}}, 0 \leq t \leq 1\right\}$. Let $E=\left\{e_{k}\right\}_{k=1}^{n}$ be a set of edges. We say $P=(V, E)$ is a polygon with vertices $V$ and edges $E$ if

(1) $\forall v \in V$, there exists exactly two distinct edges $e_{k_{1}}, e_{k_{2}} \in E$ such that $e_{k_{1}} \cap e_{k_{2}}=v$;

(2) $\forall e_{k_{1}}, e_{k_{2}}$, distinct, $e_{k_{1}} \cap e_{k_{2}}$ is either the empty set or exactly one vertex $v \in V$;

(3) The union of the edges in E forms a Jordan curve. The interior of the Jordan curve is called a face $F$ of $P$. 
The somewhat technical definition is meant to eliminate "poorly" behaved polygons which selfintersect. With this definition, polygons serve to separate $\mathbb{R}^{2}$ into a clear interior piece and an exterior piece.

Definition 2.2. The polygon $P=(V, E)$ is degenerate if it contains a vertex $v$ whose two incident edges $e_{k_{1}}, e_{k_{2}} \in E$ with $v=e_{k_{1}} \cap e_{k_{2}}$ have the same slope.

The remainder of this paper will require that $P$ is nondegenerate. Any degenerate polygon can be made nondegenerate by simply fusing the two edges which has the same slope (including the slope of infinity) into a single edge and omitting the vertex where they intersect.

Definition 2.3. A partition of a polygon $P=(V, E)$ is a planar graph $\hat{\mathcal{G}}=\{\hat{V}, \hat{E}, \hat{F}\}$ with vertices $\hat{V}$, edges $\hat{E}$ and faces $\hat{F}$ such that

(1) $V \subset \hat{V}$

(2) $\forall e \in E, \exists \hat{e}_{i_{1}}, \ldots, \hat{e}_{i_{m}} \in \hat{E}$ such that $e \subset \bigcup_{k=1}^{m} \hat{e}_{i_{k}}$

(3) $\forall \hat{v} \in \hat{V}$, there exist at least two distinct edges $\hat{e}_{k_{1}}, \hat{e}_{k_{2}} \in \hat{E}$ such that $\hat{e}_{k_{1}} \cap \hat{e}_{k_{2}}=\hat{v}$

(4) $f \in \hat{F}$ is a face if it is a polygon whose vertices and edges are elements of $\hat{V}$ and $\hat{E}$ respectively with the requirement that the interior of $F$ contains no other vertices or edges of the graph $\hat{\mathcal{G}}$. $\hat{\mathcal{G}}$.

Requirements (1) and (2) guarantee the polygon $P$ is still visible as a subset of the planar graph

Each of $\hat{V}, \hat{E}$ and $\hat{F}$ is assumed to have finite cardinality. If $|\hat{F}| \geq 2$ we say the partition is nontrivial. Finally, let $\hat{V}^{i} \subset \hat{V}$ denote the subset of interior vertices and $\hat{V}^{b} \subset \hat{V}$ denote the subset of boundary vertices.
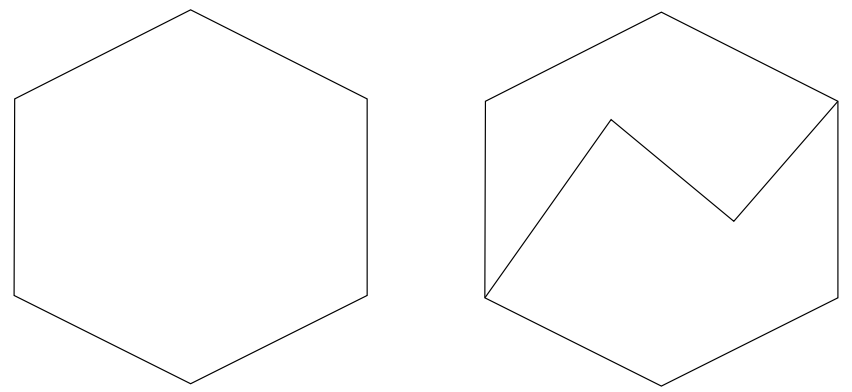

Figure 2: A hexagon can be partitioned into two nonconvex hexagons.

\subsection{Desirable Properties of Partitions}

The interest in this topic was sparked by the idea of implementing finite element schemes subordinate to polygonal partitions instead of the traditional triangulations which are a special case. As a result, the following are some basic requirements for our needs.

- Size of Underlying Partition: Based on the standard theory of spline and finite element approximation, the size of an underlying partition is required to go to zero in order to have a 

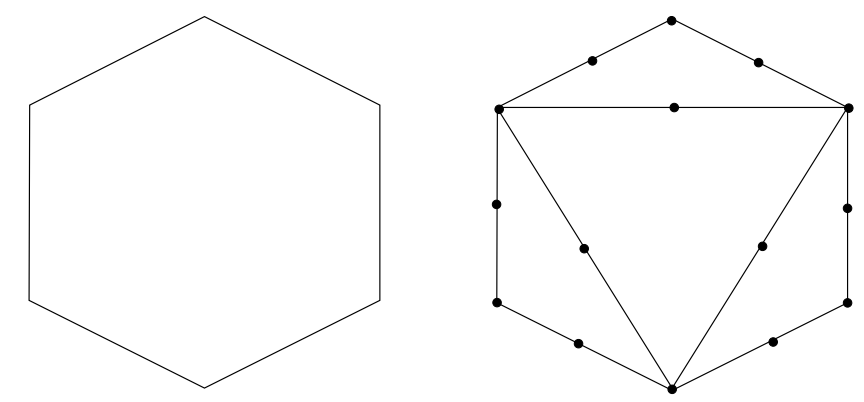

Figure 3: A hexagon can be partitioned into 4 degenerate hexagons.

good spline or finite element approximation. Refining an existing partition into a partition of the same type with finer size is a convenient approach as it can be applied repeatedly to reduce the size of underlying partitions to zero.

- Recursive refinement: In traditional finite element schemes triangulations are recursively refined until a desired numerical accuracy is reached. Recursive refinement scheme can be implemented easier and executed more efficiently than a non-recursive refinement scheme. For example, the refinement scheme for pentagonal partitions, illustrated in Figure 1 generate 6 convex pentagons which can be further refined by using the same scheme. Otherwise, if a refinement scheme has to deal with a number of cases, it increases the code complexity and the execution complexity of the refinement scheme.

- Shape regularity: In the case of triangulations, the approximation power of finite elements depends on the quality of a triangulation $\Delta$, typically measured by $\sup _{T \in \Delta} \frac{|T|}{\rho_{T}}$ where $|T|$ is the the length of the longest edge of the triangle $T$ and $\rho_{T}$ is the radius of the largest inscribed circle of $T$. The smaller this ratio, the better the approximation constant. For a discussion of shape regularity of triangulations, see [4] and [11]. As for the approximation power of finite elements defined on polygons using Generalized Barycentric Coordinates, see [8]. As a simple illustration of a poor refinement scheme, Figure 2 shows that a hexagon can be refined into two nonconvex hexagons, but the resulting hexagons have the same diameter as the original which leads to poor shape regularity. That is why we require that all polygons in the refined partition be convex as it is just a sufficient condition to avoid the scheme in Figure 2.

- Nondegeneracy: Degeneracy can give rise to trivial partitions as seen in Figure 3, where we have actually recovered a triangulation rather than some new partition scheme.

\subsection{From Geometric Shapes to Topological Surfaces}

Partitions of polygons should be regarded as geometric objects embedded in $\mathbb{R}^{2}$ where notions of convexity and angle are appropriate. The proof of our main result, however, relies on a topological argument and so our goal is to view the partition $\hat{\mathcal{G}}$ as a $\Delta$-complex or a CW complex as discussed in [9]. Then $\hat{V}$ is the set of 0 -cells, $\hat{E}$ is the set of 1 -cells and $\hat{F}$ is the set of 2-cells.

Start with two identical partitions $\hat{\mathcal{G}}$ and identify (glue) the boundary edges of each copy of $\hat{\mathcal{G}}$ to produce the $\mathrm{CW}$ complex $\mathcal{G}=\{V, E, F\}$ with 0-cells $V$, 1-cells $E$ and 2-cells $F$. Since $|\hat{F}| \geq 2$, we get $|F| \geq 4$. The result $\mathcal{G}$, which is visualized in Figure 4 , is homeomorphic to a sphere and as a result its Euler characteristic is 2. We then have the following well-known theorem (see [9], p. 146). 

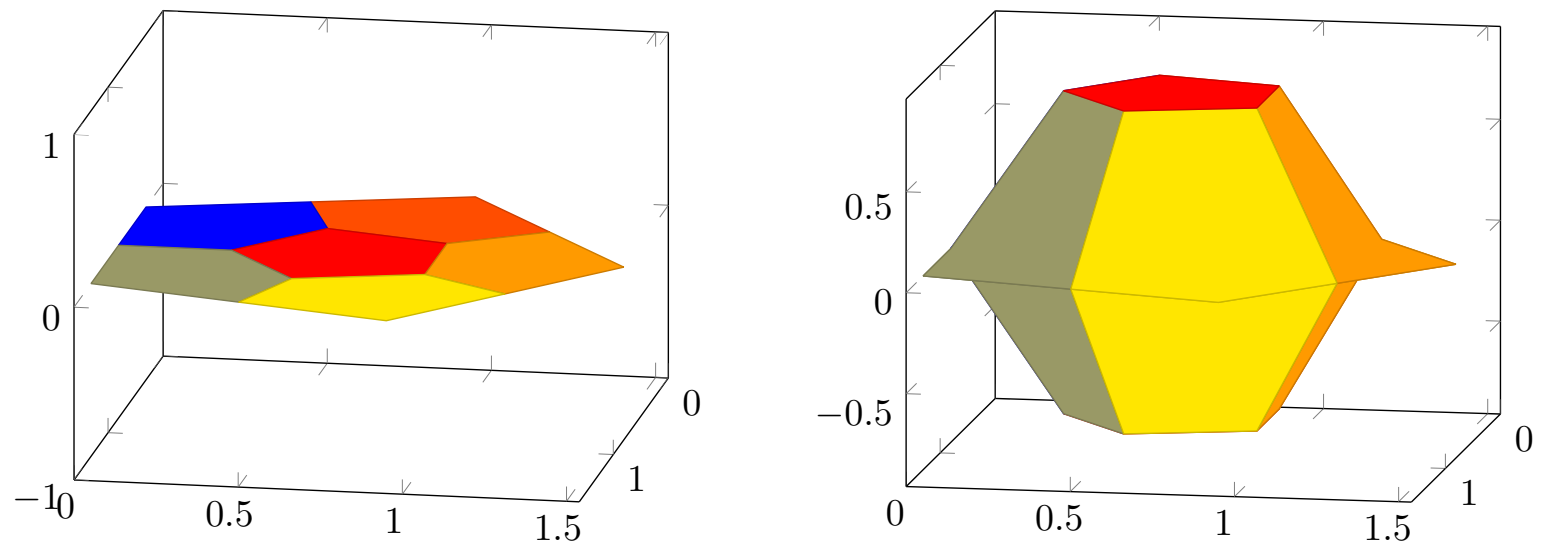

Figure 4: Left: a pentagonal partition. Right: an illustration of the $\Delta$-complex construction. The interior vertices of the partition have been lifted in the Z-direction for clarity. The construction is topological and has no associated geometrical data.

Theorem 2.1. Let $\mathcal{G}$ be a finite $C W$ complex. Then its Euler Characteristic is given by

$$
\chi(\mathcal{G})=\sum_{n}(-1)^{n} c_{n}
$$

where $c_{n}$ is the number of $n$-cells of $\mathcal{G}$.

Theorem 2.1 asserts that our object $\mathcal{G}$ obeys the formula $|V|-|E|+|F|=2$. Recall that the degree of a vertex is the number of incident edges to that vertex. We introduce the following notation.

$$
\begin{aligned}
V_{k} & =\text { number of vertices of degree } k \\
F_{k} & =\text { number of faces with } k \text { vertices }
\end{aligned}
$$

By our definition of partition, specifically assumption (3), $V_{1}=0$. That is, each vertex should have at least two incident edges.

\section{Lemma 2.1.}

$$
\begin{array}{cl}
\sum_{k=2}^{\infty} V_{k}=|V| & \sum_{k=2}^{\infty} F_{k}=|F| \\
\sum_{k=2}^{\infty} k V_{k}=2|E| & \sum_{k=2}^{\infty} k F_{k}=2|E|
\end{array}
$$

Proof. This is a simple interpretation of our notation. Note that since we assume the partition is finite, all these sums are finite.

Let us substitute the sums in Lemma 2.1 into this modified Euler characteristic equation

$$
2|V|-2|E|+2|F|=4
$$

and obtain

$$
\begin{aligned}
& \sum_{k=2}^{\infty} 2 V_{k}-\sum_{k=2}^{\infty} k V_{k}+\sum_{k=2}^{\infty} 2 F_{k}=4 \\
& \sum_{k=2}^{\infty} 2 V_{k}-\sum_{k=2}^{\infty} k F_{k}+\sum_{k=2}^{\infty} 2 F_{k}=4
\end{aligned}
$$


Add two copies of 2.1) to 2.2 to obtain the following equation:

$$
\sum_{k=2}^{\infty}(6-2 k) V_{k}+\sum_{k=2}^{\infty}(6-k) F_{k}=12 .
$$

We now state the following Lemma without proof and then proceed to the main result in this paper.

Lemma 2.2. If each interior face in $\hat{\mathcal{G}}$ is convex and nondegenerate, then $\hat{V}^{i}$ contains no vertices of degree 2.

Theorem 2.2. If $\hat{\mathcal{G}}$ is a planar graph in which every face, including the exterior face, is a nondegenerate $n$-gon for some fixed $n$ and every interior face is convex, then $n \leq 5$.

Proof. We prove the result by contradiction. Assume such a partition $\hat{\mathcal{G}}$ exists for some $n \geq 6$. Let us construct a $\mathrm{CW}$ complex $\mathcal{G}$ as discussed earlier in the section. Specialize equation (2.3) to the assumptions of this theorem, namely, $F_{k}=0$ for all $k \geq 2, k \neq n$. Thus, we have

$$
\sum_{k=2}^{\infty}(6-2 k) V_{k}+(6-n) F_{n}=12 .
$$

Let us write out the first few terms of this sum.

$$
(6-n) F_{n}+2 V_{2}-2 V_{4}-4 V_{5}-6 V_{6}-\cdots=12 .
$$

Note that the coefficient of $V_{3}$ is zero and that $V_{k} \geq 0$, so all but a few of these terms are negative. If $n \geq 6$, then $(6-n) F_{n} \leq 0$ as well. Throwing away all the negative terms from inequality 2.5 we conclude $V_{2} \geq 6$.

Claim: The only candidates for vertices of degree 2 in the $\mathrm{CW}$ complex $\mathcal{G}$ are the vertices of the original convex $n$-gon $P$. In other words,

$$
V_{2} \leq n
$$

We now prove the claim. We know from Lemma 2.2 that no interior vertices of $\hat{\mathcal{G}}$ have degree 2 , so we must look at vertices on the boundary. Any vertex $v$ on the boundary of $\hat{\mathcal{G}}$ is either a vertex of the original $n$-gon $P$ or is contained in an edge $\left(v_{i}, v_{i+1}\right)$. In the latter case, the degree of $v$ would be at least 3 since there are edges leading to $v_{i}, v_{i+1}$ and to some interior vertex. Otherwise there would exist a degenerate face, which the assumptions of the theorem forbid. Thus, the only candidates for vertices of degree 2 are the $n$ vertices of the original $n$-gon $P$, which proves the claim.

We now examine two cases regarding the number of vertices of the original polygon, each of which leads to a contradiction. Thus, the implication is that no such partition $\hat{\mathcal{G}}$ exists.

Case 1: $n \geq 7$. Since we assumed the partition is nontrivial, we know $F_{n} \geq 4$ so $(6-n) F_{n} \leq$ $4(6-n)$. Then starting with 2.5 and using 2.6 we get

$$
\begin{aligned}
12 & =(6-n) F_{n}+2 V_{2}-4 V_{4}-\ldots \\
& \leq(6-n) F_{n}+2 V_{2} \\
& \leq 4(6-n)+2 n \\
& =24-2 n .
\end{aligned}
$$

That is, $2 n \leq 12$ or $n \leq 6$ which is a contradiction. 

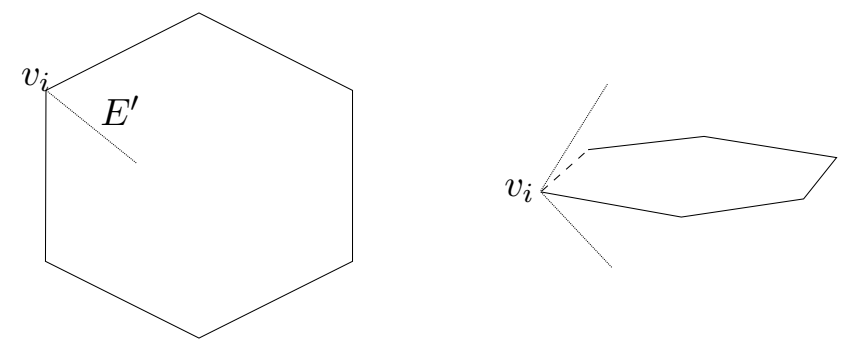

Figure 5: Left: An edge $E^{\prime}$ connects an interior point to a polygonal vertex $v_{i}$. Right: The resulting CW complex after gluing two copies of the partition along their boundaries. The degree of $v_{i}$ is at least 4.
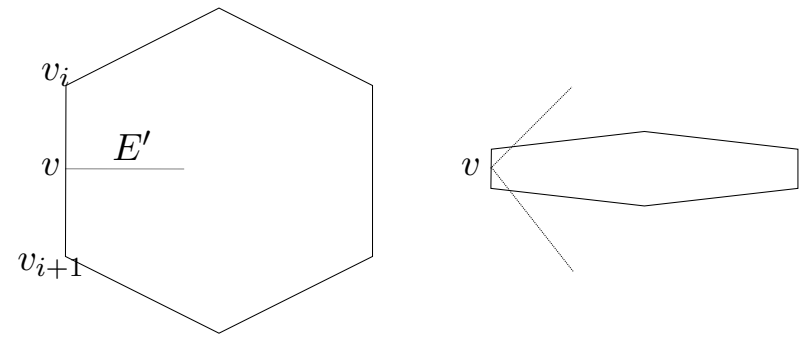

Figure 6: Left: An edge $E^{\prime}$ connects an interior point to a newly created vertex $v$ on a boundary edge. Right: The resulting CW complex after gluing two copies of the partition along their boundaries. The degree of $v$ is at least 4 .

Case 2: All that remains is to examine the interesting edge case of $n=6$, so the entire partition is composed of hexagons. In that case equation (2.5) specializes to

$$
2 V_{2}-2 V_{4}-4 V_{5}-6 V_{6}-\cdots=12
$$

It follows that $6 \leq V_{2}$. Also, from (2.6), we have $V_{2} \leq 6$ and hence, $V_{2}=6$. So we are forced to conclude that $V_{k}=0$ for all $k \geq 4$. However, we can show that at least $V_{4} \neq 0$.

Indeed, since the partition $\hat{\mathcal{G}}$ is nontrivial, there must be an interior edge $E^{\prime}$ leading from a boundary edge $E_{i}$ to the interior of the convex hull of $P$. But how is $E^{\prime}$ attached to the boundary? If it were attached to one of the original vertices $v_{i} \in P$, then the degree of $v_{i}$ in the graph $\hat{\mathcal{G}}$ would be at least 3 and upon constructing the CW complex $\mathcal{G}$, the degree of $v_{i}$ would climb to at least 4 . See Figure 5 for an illustration. As we asserted above that we need $V_{2}=6$ and the vertices $v_{i} \in P$ are the only candidates for this job, we cannot connect an interior edge to $v_{i}$.

Now suppose the edge $E^{\prime}$ connects a vertex $v$ on the boundary edge $E_{i}$ and $v \notin P$. Then the degree of that vertex in the partition $\hat{\mathcal{G}}$ is at least 3 and upon constructing the CW complex $\mathcal{G}$, the degree of $v$ would climb to at least 4 . Now we have $V_{4} \geq 1$ which contradicts (2.7). See Figure 6 for an illustration.

From Theorem 2.2, we conclude the following

Corollary 2.1. If $n \geq 6$, an $n$-gon cannot be refined by using convex, nondegenerate $n$-gons of smaller size.

Remark 2.1. The inspiration for this proof was drawn from [10]. In his book the author uses topology to treat a number of popular problems concerning polyhedra. In particular, there is a discussion on why at least twelve pentagons are necessary for the construction of a soccer ball. 


\section{Conclusion and Remarks}

Traditionally, people use triangulations for numerical solutions of PDE. Several groups of researchers have started exploring other options such as the viability of polygonal meshes for the purpose of solving PDE. We have shown in the previous section that one is not able to refine an $n$-gon using $n$-gons of smaller size for $n \geq 6$. This result is a roadblock preventing us from producing a mesh of single polygon type starting from one $n$-gon with $n \geq 6$. However, there are a few simple remedies. Let us explain a scheme for refining a hexagon by using pentagons together with a hexagon of smaller size. This is shown in Fig. 7. Similarly, we can refine an $n$-gon with $n \geq 7$ using pentagons of smaller size together with a small $n$-gon as shown in Figures 8 and 9 . These imply that if one uses polygonal meshes to numerically solve PDE, one has to construct elements or basis functions over polygons of more than one type.
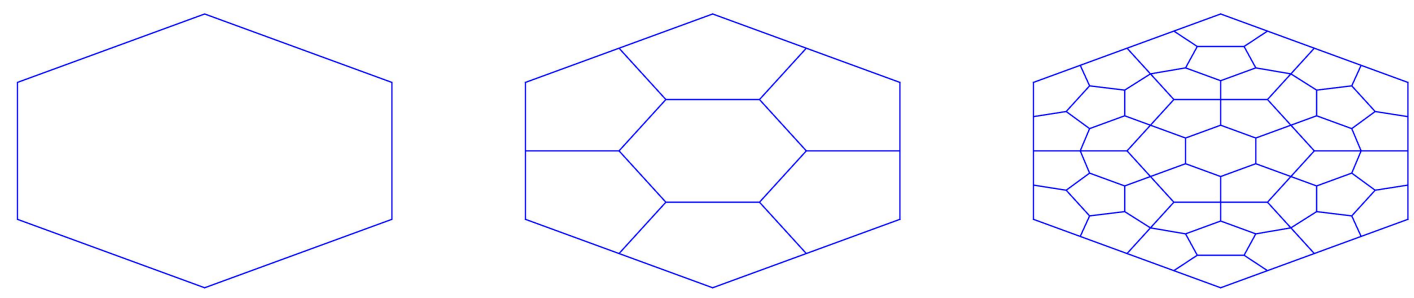

Figure 7: A hexagon and its refinement using pentagons
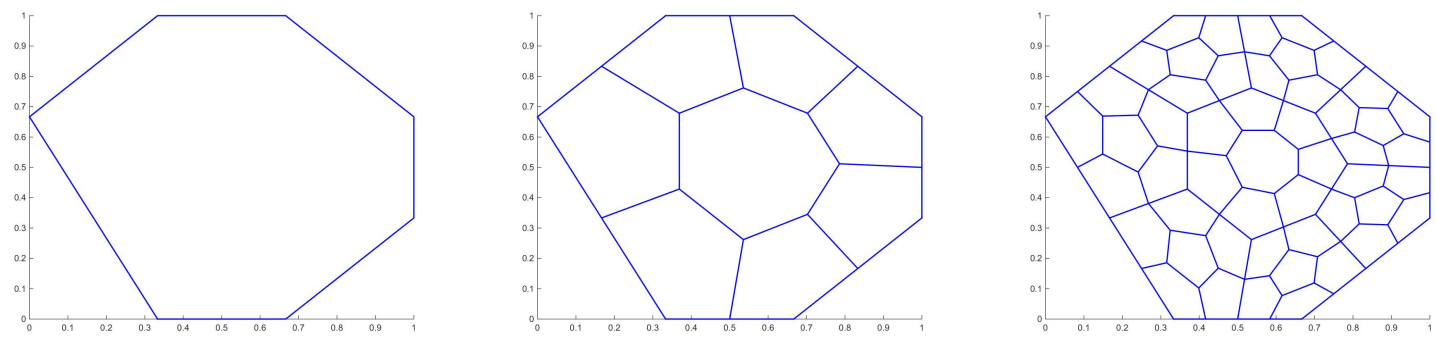

Figure 8: A heptagon and its refinement using pentagons
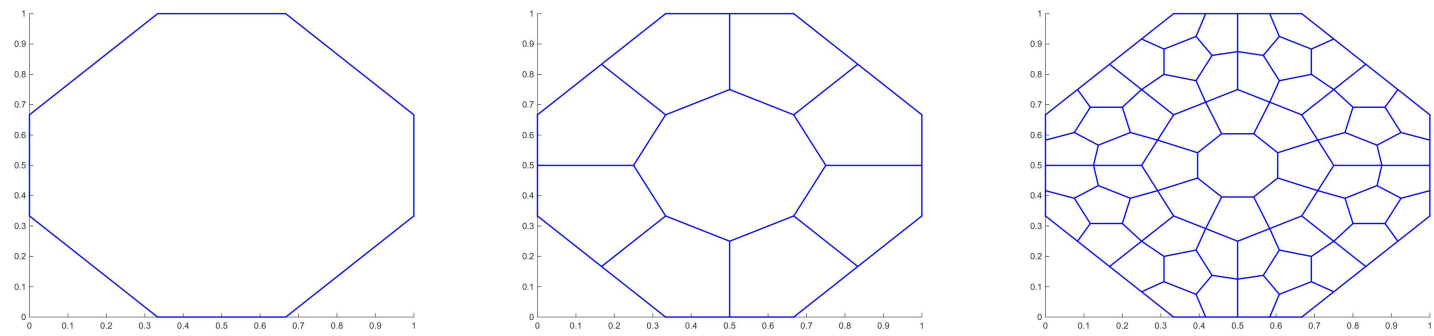

Figure 9: An octagon and its refinement using pentagons 
Indeed, we can use such a refinement of hexagons for numerical solutions of PDE, e.g. Poisson's equation. In Fig. 10, we present a numerical solution based on a modified pentagonal partition (all pentagons except for one hexagon on the top of the dome and several quadrilaterals around the boundary). The graph is produced by using the MATLAB codes based on the polygonal spline basis functions of second order constructed in [6]. See [6] for polygonal splines for numerical solution of PDE.

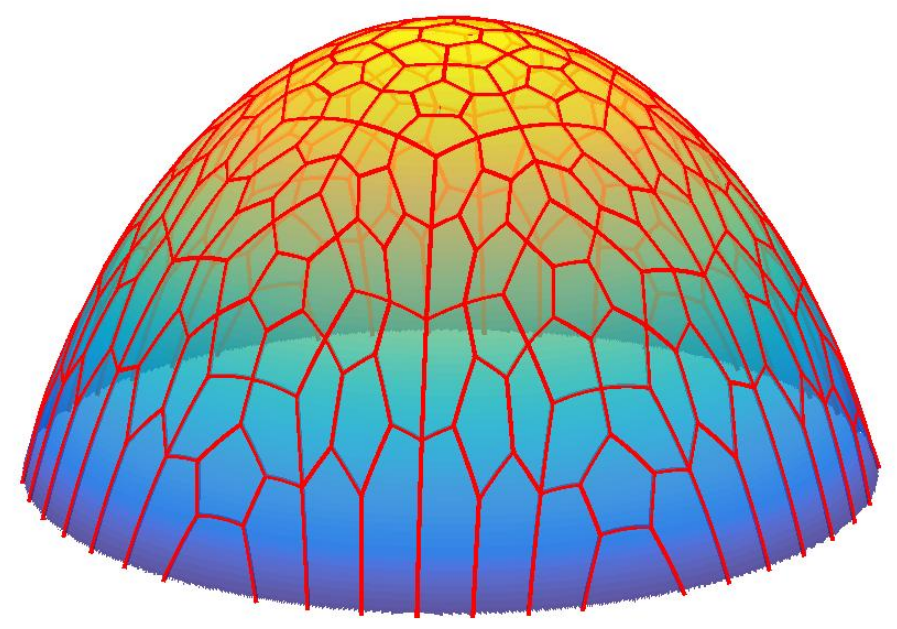

Figure 10: A numerical solution of Poisson equation using pentagons and a hexagon

Finally, let us present some remarks in order.

Remark 3.1. Of course, there are many other ways to refine an n-gon if one uses pentagons. We present a different way to refine a convex n-gon using pentagons for $n=6,7,8$ illustrated as in Fig. 11. It is clear that we can refine any convex $n$-gon for $n \geq 9$ using convex pentagons.

Remark 3.2. It is interesting to know if one can refine a triangle or quadrilateral by using pentagons of smaller sizes. To the best of our knowledge, no one knows how to do that so far. Such a refinement of triangles will be useful to partition any domain into pentagons. It is our belief that the method of proof in this paper can be adapted to answer this question.

Remark 3.3. Another open problem is to partition any polygonal domain $\Omega$ into convex pentagons. That is, $\Omega=\bigcup_{i}^{n} p_{i}$, where $p_{i}$ is a convex pentagon for each $i=1, \ldots, n$ and the intersection of any two pentagons is either the empty set or their common edge or their common vertex. It would be interesting to create such a scheme. If the open problem in Remark 3.2 can be solved, one can first use a Voronoi diagram to partition a polygon and then convert all polygons into pentagons. Indeed, for each $n$-gon, we use Remark 3.2 for $n \leq 4$ and use Remark 3.1 for $n \geq 6$. 

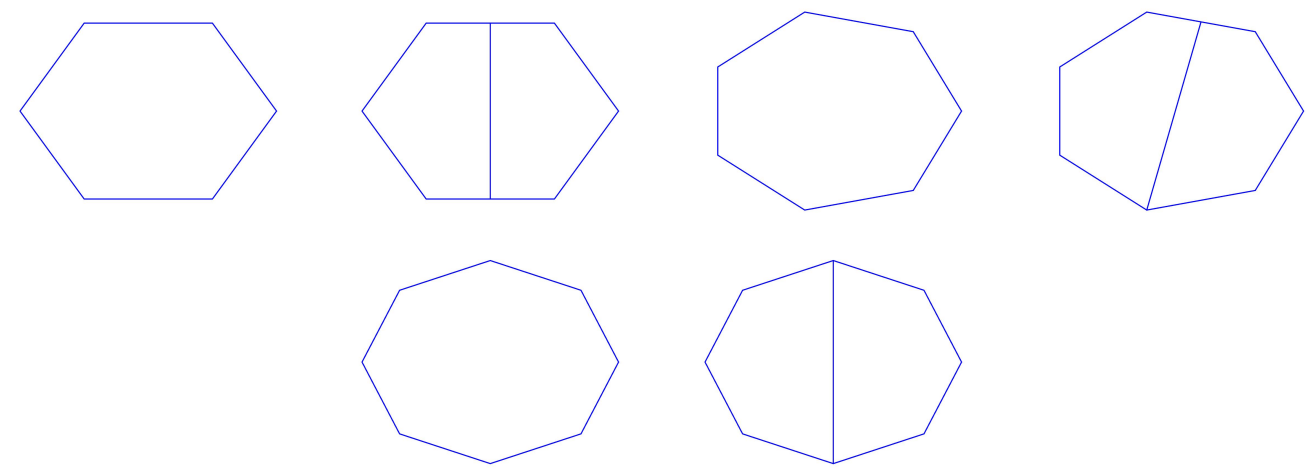

Figure 11: Subdivision of hexagon, heptagon, and octagon into pentagonal partitions

Acknowledgement: The first author would like to thank the Simons Foundation for the generosity for his Simon Collaboration grant from 2013-2018. The second author would like to thank Ernest Guico for his helpful discussion.

\section{References}

[1] G. Awanou, M. J. Lai, and P. Wenston. The multivariate spline method for scattered data fitting and numerical solutions of partial differential equations. Wavelets and splines: Athens, pages $24-74,2005$.

[2] L. Beirao da Veiga, K. Lipnikov, and G. Manzini, Arbitrary-order nodal mimetic discretizations of elliptic problems on polygonal meshes. SIAM Journal on Numerical Analysis, 49(5):1737$1760,2011$.

[3] L. Beirao da Veiga, F. Brezzi, A. Cangiani, G. Manzini, L. D. Marini, and A. Russo. Basic principles of virtual element methods. Math. Models Methods Appl. Sci., 23(1):199-214, 2013.

[4] S. C. Brenner and L. R. Scott, The mathematical theory of finite element methods, Springer Verlag, New York, 1994.

[5] M. Floater, Generalized barycentric coordinates and applications, Acta Numerica (2015), to appear.

[6] M. Floater and M. -J. Lai, Polygonal spine spaces and the numerical solution of the Poisson equation, submitted, (2015).

[7] W. H. Guo, and M. -J. Lai, Box Spline Wavelet Frames for Image Edge Analysis, SIAM Journal Imaging Sciences, vol. 6 (2013) pp. 1553-1578.

[8] A. Gillette, A. Rand, Interpolation Error Estimates for Harmonic Coordinates On Polytopes, arXiv:1504.00599 (2015).

[9] A. Hatcher, Algebraic Topology. Cambridge University Press 2002.

[10] M. Henle. A Combinatorial Introduction to Topology. Dover Publications; Reprint edition, (1994). 
[11] M. -J. Lai and L. L. Schumaker, Spline Functions on Triangulations, Cambridge Univ. Press, (2007).

[12] G. Manzini, A. Russo, and N. Sukumar, New perspectives on polygonal and polyhedral finite element methods. Math. Models Methods Appl. Sci. 24 (2014), no. 8, 1665-1699.

[13] A. Rand, A. Gillette, and C. Bajaj, Quadratic serendipity finite elements on polygons using generalized barycentric coordinates, Math. Comp. 83 (2014), 2691-2716.

[14] C. Talischi, G. H. Paulino, A. Pereira, I. F. M. Menezes, Polymesher: a general purpose mesh generator for polygonal elements written in MATLAB, Struct Multidisc Optim 45(2012) 309328.

[15] C. Wang and J. Wang, An efficient numerical scheme for the biharmonic equation by weak Galerkin finite element methods on polygonal or polyhedral meshes. Comput. Math. Appl. 68 (2014), no. 12, part B, 2314-2330 